\section{SOI: $1.1 /$ TAS $\quad$ DOI: $10.15863 /$ TAS International Scientific Journal Theoretical \& Applied Science}

p-ISSN: 2308-4944 (print) e-ISSN: 2409-0085 (online)

Year: $2018 \quad$ Issue: 03 Volume: 59

Published: $30.03 .2018 \quad$ http://T-Science.org

\section{S. U. Zhanatauov}

candidate of physics and mathematical sciences, Department «Information technologies, mathematics, physics», Associate professor, Noncommercial joint-stock company "Kazakh national agrarian university" Kazakhstan sapagtu@mail.ru

SECTION 2. Applied mathematics.

Mathematical modeling.

\title{
THE THEOREMS OF VALUES OF RELATIONSHIPS BETWEEN GROUPS OF VARIABLES
}

Abstract: The problem of finding relationships between groups of variables is central in multivariate analysis. In this work are proved the theorems of values of relationships between groups of variables. Two theorems are proved. From the Theorem 1 follows that the CCA doesn't maximize the relations necessary to use. From the Theorem 2 follows that the RA maximizes separately relations between $x$-and $v^{*}$-variables and relations between $y$-andu*-variables. Are considered Hotelling's canonical variables and Van den Wollenberg's redundancy variables. In 2 pairs groups of variables maximize a Stewart and Love's redundancy index. It is results with application only the necessary formulas for consideration CCA, RA in terms of 3 functions. In this work are highlighted the relationship between redundancy indexes in CCA and RA.

Key words: canonical correlation index, the value of relations of the biorthogonal canonical-redundancy variables.

Language: English

Citation: Zhanatauov SU (2018) THE THEOREMS OF VALUES OF RELATIONSHIPS BETWEEN GROUPS OF VARIABLES. ISJ Theoretical \& Applied Science, 03 (59): 249-256.

Soi: http://s-o-i.org/1.1/TAS-03-59-43 Doi: crossef https://dx.doi.org/10.15863/TAS.2018.03.59.43

\section{Introduction}

Correlated and standardized $\mathrm{n}$ z-variables are usually considered as one homogeneous set of states. A correlation matrix of pair coefficients $\mathrm{R}_{\mathrm{nn}}=\mathrm{R}^{\mathrm{T}}{ }_{\mathrm{nn}}=\left\{\mathrm{r}_{\mathrm{ij}}\right\}, \mathrm{i}=1, \ldots, \mathrm{n}, \mathrm{j}=1, \ldots, \mathrm{n}$ corresponds to a single set of $\mathrm{z}$-variables. Symmetric correlation matrices can have submatrices of the form of a symmetric block-diagonal matrix with blocks that differ from blocks of the Jordan block or from other species [1]. In [1], square symmetric block-diagonal n-on-n matrices consisting of 3 types of blocks were considered: from a given number of $\mathrm{n}_{1}$-by- $\mathrm{n}_{1}$-blocks $\left(\mathrm{n}_{1}>2\right)$, from 2-to-2-blocks [j: $\left.(\mathrm{j}+1)\right]$, from 1-to-1blocks ("half block" [(j-1): j] of length 1, Table 2 [1]). Elements of these blocks can be chosen randomly, for example, be random numbers with a uniform distribution law $\mathrm{P}[0,1]$. For blocks of the correlation matrix, for which the values of the elements do not exceed 1 in absolute value, and the diagonal elements are equal to 1 . In [1], the case of dividing the number $\mathrm{n}$ into 2 parts is considered: $\mathrm{n}=(\mathrm{n}-1)+1$. This partition corresponds to the partition of $n \mathrm{z}$-variables into $\mathrm{n}-1$ independent variables and to one dependent variable in the direct linear multiple regression model (DM MLRM [1,2]). If a sample $\mathrm{Z}_{1}$ of dimension $m \times(n-1)$ with known correlation matrix
$\mathrm{R}_{11}=(1 / \mathrm{m}) \mathrm{Z}^{\mathrm{T}}{ }_{1} \mathrm{Z}_{1}$ of dimension $(\mathrm{n}-1) \times(\mathrm{n}-1)$ is known, then the standardized $n$-th $\mathrm{z}$-variable with unknowns values $\mathrm{Z}_{1 \mathrm{n}}, \mathrm{Z}_{2 \mathrm{n}}, \ldots, \mathrm{Z}_{\mathrm{mn}}$ is simulating with located in the $n$-th column of the matrix $Z_{m n}=\left[Z_{1} \mid Z_{2}\right]$, $\mathrm{Z}_{2}=\left(\mathrm{Z}_{1 \mathrm{n}}, \mathrm{Z}_{2 \mathrm{n}}, \ldots, \mathrm{Z}_{\mathrm{mn}}\right)^{\mathrm{T}}$. The matrix $\mathrm{R}_{12}=(1 / \mathrm{m}) \mathrm{Z}^{\mathrm{T}}{ }_{1} \mathrm{Z}_{2}$ is the block of the matrix $R_{n n}=(1 / m) Z^{\mathrm{T}}{ }_{m n} Z_{m n}$. It is a vector of length (n-1) and its elements $r_{1 j}, r_{2 n}, r_{3 n}, \ldots, r_{n n}$ in the framework of optimization problem No. 4 [1] are given randomly, and the vector $\mathbf{B}=\mathrm{R}^{-1}{ }_{11} \mathrm{R}_{12}$ determined by it is a vector of regression coefficients. The problem of modeling the sample $Z_{m n}=\left[Z_{1} \mid Z_{2}\right]$, the correlation matrices $R_{11}, R_{12}$ for the given vector $\boldsymbol{\beta}=\left(\beta_{1}, \ldots, \beta_{\mathrm{n}-1}\right)^{\mathrm{T}}$, was called the inverse model of multidimensional regression. We will consider it in another article. In the framework of the DM MLPM model, there are already other measures of interrelations between $\mathrm{n}-1$ allocated and 1 variable, these are regression coefficients $\beta=R^{-1}{ }_{11} R_{12}$, where $\quad \mathrm{z}_{\mathrm{n}}=\mathrm{zR}^{-1}{ }_{11} \mathrm{R}_{12}=\mathrm{z} \boldsymbol{\beta}, \quad \mathrm{z}=\left(\mathrm{z}_{1}, \ldots, \mathrm{z}_{\mathrm{n}-1}\right)$. This relationship is called a regression equation showing the functional dependencies between one variable $z_{n}$ and $\mathrm{n}-1$ independent $\mathrm{z}$-variables $\mathrm{z}_{1}, \ldots, \mathrm{z}_{\mathrm{n}-1}$. These $\mathrm{n}-1$ numbers $\beta_{1}, \ldots, \beta_{\mathrm{n}-1}$ express the pair relations between the $\mathrm{n}-1 \mathrm{z}$-variables $\mathrm{Z}_{1}, \ldots, \mathrm{Z}_{\mathrm{n}-1}$ and between each of them with the variable $z_{n}$. There are many studies of the DM MLPM. Consider the case of partitioning the 
number $\mathrm{n}$ into 2 parts of the form: $\mathrm{n}=\mathrm{q}+\mathrm{p}, \mathrm{q} \geq \mathrm{p} \geq 2$. This partition corresponds to the partition of $n \mathrm{z}$ variables into $q$ independent variables and to $p$ independent variables. In this case, other multidimensional models of statistical analysis are used. The model of canonical correlations [2,3] and its alternative, the model of redundant variables $[4,5]$, enrich the previously discussed measures of the interrelations between $\mathrm{n} \mathrm{z}$-variables.

The new measures are the redundancy indexes [6]. The values of the redundancy indexes are equal to the eigenvalues of the generalized direct spectral problems (GDSP) [7,8]. In the direct model of the principal component analysis (DM PCA) and in the inverse model of the principal component analysis (IM PCA) [7-8] called the eigenvectors and eigenvalues [9-12]. In the DM PCA (in the IM PCA [7-8]) called the eigenvectors and eigenvalues direct (inverse) problem diagonalization (symmetrication) the symmetric (diagonal) matrixes [7-12]). The various linear, nonlinear functions $\left(f_{1}, f_{2}, f_{3}, f_{4}, f_{5}, f_{6}\right)$ of the elements of the spectrum $\Lambda_{\mathrm{nn}}=\operatorname{diag}\left(\lambda_{1}, \lambda_{2}, \ldots, \lambda_{\ell}, \ldots, \lambda_{\mathrm{n}}\right)$ are measures of interrelations between the $\mathrm{z}$-variables. Based on the given values of all or a part of these f-parameters, model spectra, correlation matrices, correlation matrix blocks, model $\Lambda$-samples having exactly the same correlation matrix were simulated [7-12]. Other measures of the degree of interconnections are available and implemented, if they exceed a certain threshold, they will demonstrate the "redundancy" of the manifestation of a linear connection in pairs of certain variables from the GDSP [3,13]. Below, we consider the "redundancy" of linear coupling manifestations in pairs of sets of 6 types of variables.It is known that methods of the multidimensional statistical analysis solve various problems and in each method, and in each area of its application it is necessary to overcome the difficulties. In the forecasting models using the factor analysis, the purpose is, as we will see more low, reception of high factors of determination, and also high factor loadings at values of predicted variables. This purpose satisfy few statistical methods. Each of considered below methods: CCA [2,3], RA [4,5], assumes splitting $n=q+p$ initial variables on 2 sets: $q$ $\mathrm{x}$-variables, p y-variables (they should be standardised by means of the average and standard deviations), $q \geq p$. Two sets of variables $x$ and $y$ standardized to zero mean and unit variance. It leads to use of the generalised direct spectral problems (GDSP [2,3] (обобщенная прямая спектральная задача), instead of direct problems diagonalization the symmetric matrixes (DSP- direct spectral problem [6], прямая спектральная задача). Such splitting of a vector of supervision meets in the real data. Here the real object should be characterized by two sets of properties, statistical relationship among themselves. We will more low measure values a redundancy indexes [6] steams of sets of variables in 2 methods: a canonical correlations analysis (CCA) [2,3] and in a redundancy analysis (RA ) [4,5]. Redundancy analysis (van den Wollenberg, 1977) is a popular method of multivariate analysis for analyzing the relationship between two sets of variables.

The work purpose - a finding of parities between maximum values three functions from squares of correlation coefficients between variables from two different sets. Functions are interpreted as a redundancy indexes, value of each function changes in the range of $(0,1)$ and defines average degree of expressiveness of linear interrelation between two sets of variables [2]. In terms of these functions average shares of dispersions of variables of each of two sets of the initial variables, explained by their correlations with variables from other set are investigated.

Let's consider 3 pairs (from 6) sets of the initial, canonical, redundancy variables received in CCA, RA. When transforming using the RA of canonical variables, we obtain new canonical-redundancy variables. A pair of new variables consists of $2 p=p$ $+p$ variables. The found formulas redundancy are necessary for a finding of pairs sets of variables (or factors). The found redundancy formulas are effective when predicting the values of some input variables from the values of other variables. We will state results with reduction only the necessary formulas for consideration CCA, RA in terms of 3 functions.

\section{The redundancy formulas of the non- symmetrical redundancy of the $x$ - and $y$ - variables in biorthogonal canonical correlation analysis}

The essence of CCA consists in a finding as much as possible correlated among themselves $\mathrm{u}$ and v-variables, i. e. two linear combinations initial $\mathrm{x}$ - (with factors $\mathrm{a}_{1 \mathrm{j}}, \ldots, \mathrm{a}_{\mathrm{qj}}$ at $\mathrm{j}$-th u-variable) and $\mathrm{u}$ variables (with factors $b_{1 j}, \ldots, b_{p j}$ at $j$-th v-variables), named canonical $\mathrm{u}-$ and $\mathrm{v}$ - variables, satisfying to restrictions:

$$
\begin{gathered}
\mathrm{U}_{\mathrm{mp}}=\mathrm{Z}_{1} \mathrm{~A}_{\mathrm{qp}}, \mathrm{V}_{\mathrm{mp}}=\mathrm{Z}_{2} \mathrm{~B}_{\mathrm{pp}},(1 / \mathrm{m}) \mathrm{U}^{\mathrm{T}} \mathrm{U}=\mathrm{I}_{\mathrm{pp}}, \\
(1 / \mathrm{m}) \mathrm{V}^{\mathrm{T}} \mathrm{V}=\mathrm{I}_{\mathrm{pp}},(1 \mathrm{~m}) \mathrm{U}^{\mathrm{T}} \mathrm{V}=\Lambda_{\mathrm{pp}} \\
\mathrm{R}_{12}=(1 / \mathrm{m}) \mathrm{Z}_{1}^{\mathrm{T}} \mathrm{Z}_{2}, \mathrm{R}_{21}=(1 / \mathrm{m}) \mathrm{Z}_{2}^{\mathrm{T}} \mathrm{Z}_{1}, \\
\mathrm{R}_{11}=(1 / \mathrm{m}) \mathrm{Z}_{1}^{\mathrm{T}} \mathrm{Z}_{1}, \mathrm{R}_{22}=(1 / \mathrm{m}) \mathrm{Z}_{2}^{\mathrm{T}} \mathrm{Z}_{2},
\end{gathered}
$$

Vectors $\mathbf{a}_{\mathrm{j}}=\left(\mathrm{a}_{1 \mathrm{j}}, \ldots, \mathrm{a}_{\mathrm{qij}}\right)^{\mathrm{T}}, \mathbf{b}_{\mathrm{j}}=\left(\mathrm{b}_{1 \mathrm{j}}, \ldots, \mathrm{b}_{\mathrm{qj}}\right)^{\mathrm{T}}$ and factors of canonical correlations $\mathbf{r}_{\mathrm{uv}}=(1 / m) \sum_{i=1}^{m} \boldsymbol{u}_{i j} v_{i j}=1, \mathrm{u}=\mathrm{u}_{\mathrm{j}}$, $\mathrm{v}=\mathrm{v}_{\mathrm{j}}, \mathrm{j}=1, \ldots, \mathrm{p}$, are from mathematical equations of maxima of these correlations

$\mathbf{r}_{\mathrm{uv}}=\mathbf{a R} \mathbf{R}_{12} \mathbf{b} \rightarrow \max _{a, b}$, under equations of (1), where $\mathbf{a}$, $\mathbf{b}-\mathbf{j}$ - th eigen vectors from GDSP (3). 
Necessary conditions of maxima are [1] matrix equations.

$$
\begin{aligned}
& R_{12} b_{j}=\mu_{j} R_{11} a_{j} \\
& R_{21} a_{j}=v_{j} R_{22} b_{j}
\end{aligned}
$$

As $\mathbf{a}_{\mathrm{j}}{ }^{\mathrm{T}} \mathrm{R}_{11} \mathbf{a}_{\mathrm{j}}=1, \mathrm{~b}_{\mathrm{j}}^{\mathrm{T}} \mathrm{R}_{22} \mathrm{~b}_{\mathrm{j}}=1$, from these matrix equations follows [1] that $\mu_{j}=v_{j}=r_{u v}, u=u_{j}, v=v_{j}$. Then matrix equations (2) can be written down in a kind

$$
\begin{aligned}
& \left(R_{12} R^{-1}{ }_{22} R_{21}-\mu^{2}{ }_{j} R_{11}\right) a_{j}=0, \\
& \left(R_{21} R^{-1}{ }_{11} R_{12}-v^{2}{ }_{j} R_{22}\right) b_{j}=0 .
\end{aligned}
$$

As $R_{n n}$ it is positively defined $\left(R_{n n} \neq I_{n n}, R_{n n}>0\right)$ there are unique 2 matrixes $A_{q p},=\left[\mathbf{a}_{j}|\ldots| \mathbf{a}_{\mathrm{p}}\right]$, $B_{p p}=\left[b_{j}|\ldots| \mathbf{b}_{\mathrm{p}}\right]$ eigen vectors which are calculated by the solve of one of two GDSP (3). If it is found a vector $\mathbf{a}_{j}$, then $\mathbf{b}_{j}=v^{-1}{ }_{j} R^{-1}{ }_{22} R_{21} \mathbf{a}_{j}$, if it is found $\mathbf{b}_{j}$, then $\mathbf{a}_{\mathrm{j}}=\mu^{-1}{ }_{j} \mathrm{R}^{-1}{ }_{11} \mathbf{R}_{12} \mathbf{b}_{\mathrm{j}}$. Here $\mathrm{p}$ positive eigen values $\mu^{2} 1, \ldots, \mu^{2}$ are equal to eigen values $v^{2}, \ldots, v_{p}^{2}$ and are equal to squares of factors of canonical correlations $\mathrm{r}^{2}{ }_{\mathrm{uv}} \equiv \lambda_{\mathrm{j}}^{2}=\mu_{\mathrm{j}}^{2}=\mathrm{v}_{\mathrm{j}}^{2}$, and, corresponding eigen vectors $\mathbf{a}_{\mathrm{j}}=\left(\mathrm{a}_{1 \mathrm{j}}, \ldots, \mathrm{a}_{\mathrm{qj}}\right)^{\mathrm{T}}, \quad \mathbf{b}_{\mathrm{j}}=\left(\mathrm{b}_{1 \mathrm{j}}, \ldots, \mathrm{b}_{\mathrm{qj}}\right)^{\mathrm{T}}$ are located on columns of matrixes $\mathrm{A}_{\mathrm{qp}}, \mathrm{B}_{\mathrm{pp}}$. As to a maximum $\mathrm{r}_{\mathrm{uv}}$ there corresponds a maximum $r^{2}$ uv parities received in CCA are fair and at maximisation of function $\varphi_{1}$ $\left(r^{2}{ }_{u v}\right)=r^{2}$ uv under conditions (1). The parities connecting matrixes of canonical variables $\mathrm{U}_{\mathrm{mp}}, \mathrm{V}_{\mathrm{mp}}$, canonical loadings - $\mathrm{A}_{\mathrm{qp}}, \mathrm{B}_{\mathrm{pp}}$, initial variables - $\mathrm{Z}_{1}$ (dimensions $\mathrm{m} \times \mathrm{q}$ ), $\quad \mathrm{Z}_{2}$ (dimensions $\mathrm{m} \times \mathrm{p}$ ), intragroup correlations $\mathrm{R}_{11}, \mathrm{R}_{22}$, intergroup correlations - $\mathrm{R}_{12}$, functions corresponding to the maximum value $\varphi_{1}$ :

$$
\varphi_{1}\left(\mathrm{r}_{\mathrm{uv}}^{2}\right)=\mathrm{r}_{\mathrm{uv}}^{2}=\max _{1} \text {, look like: }
$$

$\mathrm{U}=\mathrm{Z}_{1} \mathrm{~A}, \mathrm{~V}=\mathrm{Z}_{2} \mathrm{~B},(1 / \mathrm{m}) \mathrm{U}^{\mathrm{T}} \mathrm{U}=\mathrm{A}^{\mathrm{T}} \mathrm{R}_{11} \mathrm{~A}=\mathrm{I}_{\mathrm{pp}}$, $(1 / \mathrm{m}) \mathrm{V}^{\mathrm{T}} \mathrm{V}=\mathrm{B}^{\mathrm{T}} \mathrm{R}_{22} \mathrm{~B}=\mathrm{I}_{\mathrm{pp}}$

$(1 / \mathrm{m}) \mathrm{UTV}=\mathrm{A}^{\mathrm{T}} \mathrm{R}_{12} \mathrm{~B}=\Lambda_{\mathrm{pp}}, \Lambda_{\mathrm{pp}}=\operatorname{diag}\left(\lambda_{1}, \ldots, \lambda_{\mathrm{p}}\right)$.

In addition to formulas of CCA Stewart D., Love W. [2] have offered the formula of the redundancy index, of the $\mathrm{u}$-variables (v-variables), equal to an average explained dispersion $y$-variables (x-variables) in the presence of one canonical $u$ variable (v-variable). Formulas of these indexes in CCA in our designations have an appearance accordingly

$$
\begin{aligned}
& R_{\mathrm{p}}(\mathrm{Y}, \mathrm{u})=(1 / \mathrm{p}) \mathrm{r}^{\mathrm{T}}{ }_{\mathrm{Yu}}^{\mathrm{r}} \mathrm{Yu}=(1 / \mathrm{p}) \sum_{i=1}^{p} r_{y_{i} u}^{2} \equiv \varphi_{2}\left(\mathrm{r}^{2} \mathrm{Yu}\right), \\
& R_{\mathrm{q}}(\mathrm{X}, \mathrm{v})=(1 / \mathrm{q}) \mathrm{r}^{\mathrm{T}}{ }_{\mathrm{Xv}}^{\mathrm{r} \mathrm{Xv}_{\mathrm{v}}}=(1 / \mathrm{q}) \sum_{i=1}^{q} \boldsymbol{r}_{x_{i} v}^{2} \equiv \varphi_{3}\left(\mathrm{r}^{2} \mathrm{Xv}\right),
\end{aligned}
$$

Where $\quad \mathbf{r}_{\mathrm{Yu}}=\mathrm{R}_{21} \mathbf{a}, \quad \mathbf{r}_{\mathrm{Xv}}=\mathrm{R}_{12} \mathbf{b}, \quad$ vectors $\mathbf{a}=\left(\mathrm{a}_{1}, \ldots, \mathrm{a}_{\mathrm{q}}\right)^{\mathrm{T}}, \quad \mathbf{b}=\left(\mathrm{b}_{1}, \ldots, \mathrm{b}_{\mathrm{p}}\right)^{\mathrm{T}}$-j-th eigen vectors (columns of matrixes $\mathrm{A}_{\mathrm{qp}}, \mathrm{B}_{\mathrm{pp}}$ ), corresponding to $\mathrm{j}$-th eigen values $\lambda^{2}$, where $\varphi_{2}, \varphi_{3}$ - defined above functions. The following definitions are similar given in [3]. We are compelled to simplify a type of a formula. This formula designates a square of coefficient of pair correlation $\mathrm{r}^{2}$ between $\mathrm{j}$-th $\mathrm{y}$ variable and $i$ - th a $u$-variable, i. e. $r^{2}$ with the bottom $\mathrm{y}_{\mathrm{j}} \mathrm{u}_{\mathrm{i}}$ indexes. It is inconvenient at the formulation of exact definitions. Therefore, for convenience we will designate this formula so: $\mathrm{r}^{2}\left(\mathrm{y}_{\mathrm{j}}, \mathrm{u}_{\mathrm{i}}\right)$, and a formula
$\mathbf{r}^{\mathrm{T}}{ }_{\mathrm{Yu}} \mathbf{r}_{\mathrm{Yu}}$ for i- th u-variable we will write in the form $\mathrm{r}^{2}\left(\mathrm{Y}, \mathrm{u}_{\mathrm{j}}\right)$. Similarly: $\mathbf{r}_{\mathrm{Xv}}^{\mathrm{T}} \mathbf{r}_{\mathrm{Xv}}$ for $\mathrm{i}$ - th v-variable - in the form $\mathrm{r}^{2}\left(\mathrm{X}, \mathrm{v}_{\mathrm{j}}\right)$.

Definition 1. Total redundancy of all $p y-$ variables at presence $\mathrm{p} \mathrm{u}$-variables is equal

$$
R_{\mathrm{p}}(\mathrm{Y}, \mathrm{U})=\sum_{i=1}^{p} R_{\mathrm{p}}\left(\mathrm{Y}, \mathrm{u}_{\mathrm{i}}\right)=\sum_{i=1}^{p} \varphi_{2}\left(\mathbf{r}^{2}\left(\mathrm{Y}, \mathrm{u}_{\mathrm{i}}\right)\right) .
$$

Definition 2. Total redundancy of all $q \mathrm{x}-$ variables at presence $\mathrm{p} v$-variables is equal:

$$
R_{\mathrm{q}}(\mathrm{X}, \mathrm{V})=\sum_{i=1}^{p} R_{\mathrm{q}}(\mathrm{X}, \mathrm{v})=\sum_{i=1}^{p} \varphi_{3}\left(\mathbf{r}^{2}\left(\mathrm{X}, \mathrm{v}_{\mathrm{i}}\right)\right),
$$

If to use necessary conditions (2) maxima in CCA now formulas redundancies $\mathrm{R}_{\mathrm{p}}(\mathrm{Y}, \mathrm{u})$ and $\mathrm{R}_{\mathrm{q}}(\mathrm{X}, \mathrm{v})$ become [2]:

$$
\varphi_{2}\left(\mathbf{r}^{2} \mathrm{Yu}\right)=R_{\mathrm{p}}(\mathrm{Y}, \mathrm{u})=(1 / \mathrm{p}) \mathbf{r}^{\mathrm{T}}{ }_{\mathrm{Yu}} \mathbf{r}_{\mathrm{Yu}}=(1 / \mathrm{p})\left(\mathrm{R}_{21} \mathbf{a}\right)^{\mathrm{T}}\left(\mathrm{R}_{21} \mathbf{a}\right)=
$$

$(1 / \mathrm{p})\left(v \mathrm{R}_{22} \mathbf{b}\right)^{\mathrm{T}}\left(v \mathrm{R}_{22} \mathbf{b}\right)=(1 / \mathrm{p}) v^{2} \mathbf{r}^{\mathrm{T}} \mathrm{Yv}_{\mathrm{v}} \mathbf{r}_{\mathrm{Yv}}=v^{2} R_{\mathrm{p}}(\mathrm{Y}, \mathrm{v})=$ $\varphi_{1}\left(\mathbf{r}_{\mathrm{uv}}^{2}\right) \times \varphi_{2}\left(\mathbf{r}^{2} \mathrm{Yv}_{\mathrm{v}}\right), \varphi_{3}\left(\mathbf{r}_{\mathrm{Xv}}^{2}\right)=$

$$
R_{\mathrm{q}}(\mathrm{X}, \mathrm{v})=(1 / \mathrm{q}) \mathbf{r}^{\mathrm{T}}{ }_{\mathrm{Xv}} \mathbf{r}_{\mathrm{Xv}}=(1 / \mathrm{q})\left(\mathrm{R}_{12} \mathbf{b}\right)^{\mathrm{T}}\left(\mathrm{R}_{12} \mathbf{b}\right)=
$$$$
(1 / \mathrm{q})\left(\mu \mathrm{R}_{11} \mathbf{a}\right)^{\mathrm{T}}\left(\mu \mathrm{R}_{11} \mathbf{a}\right)=
$$$$
=(1 / \mathrm{q}) \mu^{2} \mathbf{r}^{\mathrm{T}}{ }_{\mathrm{Xu}} \mathbf{r}_{\mathrm{Xu}}=\mu^{2} R_{\mathrm{q}}(\mathrm{X}, \mathrm{u})=\varphi_{1}\left(\mathbf{r}^{2}{ }_{\mathrm{uv}}\right) \times \varphi_{3}\left(\mathbf{r}^{2}{ }_{\mathrm{Xu}}\right) \text {. }
$$

Thus, we have equations:

$$
\begin{gathered}
\varphi_{2}\left(\mathbf{r}^{2} \mathrm{Yu}\right)=\varphi_{1}\left(\mathbf{r}^{2} \text { uv }\right) \times \varphi_{2}\left(\mathbf{r}^{2}{ }_{\mathrm{Yv}}\right), \\
\varphi_{3}\left(\mathbf{r}^{2} \mathrm{Xv}\right)=\varphi_{1}\left(\mathbf{r}_{\text {uv }}^{2}\right) \times \varphi_{3}\left(\mathbf{r}^{2} \mathrm{Xu}\right)
\end{gathered}
$$

As $\varphi_{1}\left(\mathbf{r}^{2}{ }_{\text {uv }}\right)<1$, inequalities take place

$$
\varphi_{2}\left(\mathbf{r}^{2} \mathrm{Yu}\right)<\varphi_{2}\left(\mathbf{r}^{2} \mathrm{Yv}_{\mathrm{v}}\right), \varphi_{3}\left(\mathbf{r}^{2} \mathrm{Xv}\right)<\varphi_{3}\left(\mathbf{r}^{2} \mathrm{Xu}\right) \text {. }
$$

Lower will formulas $\varphi_{2}\left(\mathbf{r}^{2} \mathrm{Yv}\right)=1 / \mathrm{p}, \varphi_{3}\left(\mathbf{r}^{2} \mathrm{Xu}\right)=1 / \mathrm{q}$ are received.

Lemma. In CCA an average dispersion of all canonical $y$-variables ( $\mathrm{x}$-variables), is explained $\mathrm{i}$-th one u-variables (v-variable), less than the average dispersion of all $\mathrm{y}$-variables (x-variables) explained by one v-variable (u-variable):

$$
\begin{aligned}
\varphi_{3}\left(\mathbf{r}_{\mathrm{Yv}}^{2}\right) & =\varphi_{1}\left(\mathbf{r}_{\text {uv }}^{2}\right) \times \varphi_{3}\left(\mathbf{r}^{2}{ }_{\mathrm{Yu}}\right)=(1 / \mathrm{p}) \lambda^{2}, \\
\varphi_{3}\left(\mathbf{r}_{\mathrm{Xv}}^{2}\right) & =\varphi_{1}\left(\mathbf{r}_{\mathrm{uv}}^{2}\right) \times \varphi_{3}\left(\mathbf{r}^{2}{ }_{\mathrm{Xu}}\right)=(1 / \mathrm{q}) \lambda^{2},
\end{aligned}
$$

where $\lambda$ - $\mathrm{j}$-th coefficient of canonical correlation.

In the Lemma of the formula (4) redundancy of the canonical $\mathrm{u}$-variables interpret as follows. The value $v^{2}$ is proportional to the explained dispersion of an canonical $\mathrm{u}$-variable provided that there is a vvariable correlating with it the second factor of the formula (4) is proportional to the average explained dispersion of all $y$-variables under a similar condition.

\section{The redundancy formulas of the non- symmetrical redundancy \\ of the $x$ - and $y$-variables in non biorthogonal redundancy variables analysis} number

In CCA product of these two factors to equally

$$
\varphi_{2}\left(\mathbf{r}^{2} \mathrm{Yu}\right)=(1 / \mathrm{p}) \mathbf{r}^{\mathrm{T}}{ }_{\mathrm{u}} \mathbf{r}_{\mathrm{Yu}}=(1 / \mathrm{p}) \sum_{i=1}^{p} r_{y_{i}{ }^{u}}^{2} \equiv R_{\mathrm{p}}(\mathrm{Y}, \mathrm{u}),
$$

which is not maximised in itself, but it is proportional to the maximum value $\varphi_{1}\left(\mathrm{r}^{2} \mathrm{uv}\right)$. In work 
[5] vector $\mathbf{r}_{\mathrm{Yu}}=\mathrm{R}_{21} \mathbf{a}$ is designated in a matrix form $(1 / \mathrm{m}) \mathrm{Z}_{2}^{\mathrm{T}} \mathrm{U}=(1 / \mathrm{m}) \mathrm{Z}^{\mathrm{T}}{ }_{2} \mathrm{Z}_{1} \mathbf{a}=\mathrm{R}_{21} \mathbf{a}$, hence we have $\left.\varphi_{2}\left(r^{2}{ }_{Y u}\right)=(1 / p) r^{\mathrm{T}}{ }_{Y u} r_{Y u}=\left(R_{21} a\right)^{\mathrm{T}} R_{21} a\right)=\mathbf{a}^{\mathrm{T}} \mathrm{R}_{12} \mathrm{R}_{21} \mathbf{a}=$ $=(1 / p)\left(v R_{22} \mathbf{b}\right)^{\mathrm{T}}\left(v \mathrm{R}_{22} \mathbf{b}\right)=(1 / \mathrm{p})\left(\mathbf{b}^{\mathrm{T}} \mathrm{R}^{2}{ }_{22} \mathbf{b}\right) v^{2}=$ $=(1 / \mathrm{p}) v^{2} \mathbf{r}^{\mathrm{T}}{ }_{\mathrm{Yv}} \mathbf{r}_{\mathrm{Yv}}=v^{2} R_{\mathrm{p}}(\mathrm{Y}, \mathrm{v})=v^{2}(1 / \mathrm{p})=\max _{1} \times \max _{3}$ If a vector $\mathbf{r}_{\mathrm{Xv}}$ equal $\mathbf{r}_{\mathrm{Xv}}=\mathrm{R}_{12} \mathbf{b}$, that

$$
\begin{gathered}
\varphi_{2}\left(\mathbf{r}^{2} \mathrm{Yu}\right)=R_{\mathrm{p}}(\mathrm{Y}, \mathrm{u})=(1 / \mathrm{p}) \mathbf{r}^{\mathrm{T}} \mathrm{Yu}_{\mathbf{Y u}}=(1 / \mathrm{p})\left(\mathrm{R}_{21} \mathbf{a}\right)^{\mathrm{T}}\left(\mathrm{R}_{21} \mathbf{a}\right)= \\
=(1 / \mathrm{p})\left(\mathrm{v} \mathrm{R}_{22} \mathbf{b}\right)^{\mathrm{T}}\left(v \mathrm{R}_{22} \mathbf{b}\right)=(1 / \mathrm{p}) v^{2} \mathbf{r}^{\mathrm{T}} \mathrm{Yv}_{\mathrm{Y}} \mathbf{r}_{\mathrm{Yv}}=v^{2} R_{\mathrm{p}}(\mathrm{Y}, \mathrm{v}) \\
=\varphi_{1}\left(\mathbf{r}_{\mathrm{uv}}^{2}\right) \times \varphi_{2}\left(\mathbf{r}^{2} \mathrm{Yv}_{\mathrm{v}}\right) .
\end{gathered}
$$$$
\text { Similarly we have }
$$$$
\varphi_{3}\left(\mathrm{r}^{2}{ }_{\mathrm{Xv}}\right)=R_{\mathrm{q}}(\mathrm{X}, \mathrm{v})=(1 / \mathrm{q}) \mathrm{r}^{\mathrm{T}} \mathrm{Xv}_{\mathrm{Xv}}^{\mathrm{r}}=(1 / \mathrm{q})\left(\mathrm{R}_{12} \mathrm{~b}\right)^{\mathrm{T}}\left(\mathrm{R}_{12} \mathrm{~b}\right)=
$$$$
=(1 / \mathrm{q})\left(\mu \mathrm{R}_{11} \mathrm{a}\right)^{\mathrm{T}}\left(\mu \mathrm{R}_{11} \mathrm{a}\right)=(1 / \mathrm{q}) \mu^{2} \mathrm{r}^{\mathrm{T}}{ }_{\mathrm{Xu}}^{\mathrm{r}} \mathrm{Xu}=\mu^{2} R_{\mathrm{q}}(\mathrm{X}, \mathrm{u})=
$$$$
=\varphi_{1}\left(\mathbf{r}_{\mathrm{uv}}^{2}\right) \times \varphi_{3}\left(\mathbf{r}^{2} \mathrm{Xu}\right)=\max _{1} \times \max _{2} \text {. }
$$

In CCA value of function $\varphi_{3}\left(\mathbf{r}^{2} X_{v}\right)$ as product $\varphi_{3}\left(\mathbf{r}^{2} \mathrm{Xv}\right)=\varphi_{1}\left(\mathbf{r}_{\mathrm{uv}}^{2}\right) \times \varphi_{3}\left(\mathbf{r}^{2} \mathrm{Xu}\right)$ two values $\varphi_{1}\left(\mathbf{r}^{2}{ }_{\mathrm{uv}}\right)$ and $\varphi_{3}\left(\mathbf{r}^{2} \mathrm{Xu}\right)$, is equal to value $R_{\mathrm{q}}(\mathrm{X}, \mathrm{v})$ :

$$
\varphi_{3}\left(\mathbf{r}^{2} \mathrm{Xv}\right)=(1 / \mathrm{q}) \mathbf{r}^{\mathrm{T}}{ }_{\mathrm{Xv}}{ }^{\mathrm{r}} \mathrm{Xv}=(1 / \mathrm{q}) \sum_{i=1}^{p} r_{y_{i}{ }^{u}}^{2}=R_{\mathrm{q}}(\mathrm{X}, \mathrm{v}) .
$$

It would be desirable to maximise specified to redundancy in itself, i.e. separately. Differently, it is necessary to find such linear combinations from each set of variables which would maximise the average explained dispersion of variables of other set.

$$
\begin{aligned}
& R_{\mathrm{p}}\left(\mathrm{Y}, \mathrm{u}^{*}\right)=(1 / \mathrm{p}) \sum_{i=1}^{p} r_{y_{i} u^{*}}^{2} \equiv \varphi_{2}\left(\mathrm{r}^{2} \mathrm{Yu}^{*}\right) \rightarrow \operatorname{maX}_{a^{*}} \\
& R_{\mathrm{q}}\left(\mathrm{X}, \mathrm{v}^{*}\right)=(1 / \mathrm{q}) \sum_{i=1}^{q} r_{x_{i}}^{2} \nu^{*} \equiv \varphi_{3}\left(\mathrm{r}^{2} \mathrm{Xv}^{*}\right) \rightarrow \max _{b^{*}}
\end{aligned}
$$

Thus $\varphi_{2}\left(\mathrm{r}^{2} \mathrm{Yu}^{*}\right), \varphi_{3}\left(\mathbf{r}^{2} \mathrm{Xv}^{*}\right)$ it is desirable to maximise functions independently from each other. It is made in work [2], where for new so-called redundancy $\mathrm{u}^{*}$ - and $\mathrm{v}^{*}$-variables equalities (4) any more are not satisfied. A method of redundancy variables (RA ) Van den Vollenberg-s A.L. [4] is alternative to CCA $\mathrm{H}$. Hotelling-s [2] for other interrelations, than in CCA are maximized. The essence of RA [4] consists in a finding of linear combinations of redundancy $\mathrm{u}^{*}$-variables $\mathrm{u}^{*}{ }_{\mathrm{ij}}=$ $\sum_{i=1}^{q} Z_{j} \boldsymbol{A}^{*}$ of initial x-variables, as much as possible correlated with y-variables, and in a finding of linear combinations of redundancy $\mathrm{v}^{*}$-variables $\mathrm{v}^{*}{ }_{\mathrm{ij}}=$ $\sum_{k=1}^{p} Z_{i(k+\check{u}} b_{k j}, \mathrm{i}=1, \ldots, \mathrm{m} ; \mathrm{j}=1, \ldots, \mathrm{n}$, it is maximum is correlated the about an outcome $\mathrm{j}$-th $\mathrm{x}$-variables. Vectors $\mathbf{a}^{*}=\left(\mathbf{a}^{*}{ }_{1 j}, \ldots, a^{*}{ }_{\mathrm{q} j}\right)^{\mathrm{T}}, \mathbf{b}_{\mathrm{j}}{ }_{\mathrm{j}}=\left(\mathrm{b}^{*}{ }_{1 \mathrm{j}}, \ldots, \mathrm{b}_{\mathrm{pj}}\right)^{\mathrm{T}}-\mathrm{j}-$ th eigen vectors from GDSP (6), (7) are from maximum conditions, accordingly, functions

$$
\varphi_{2}\left(\mathrm{r}^{2} \mathrm{Yu}^{*}\right)=(1 / \mathrm{p}) \sum_{i=1}^{p} r_{y_{i}}^{2} u^{*} \rightarrow \max _{a^{*}}
$$

$$
\varphi_{3}\left(\mathrm{r}^{2} \mathrm{Xv}^{*}\right)=(1 / \mathrm{q}) \sum_{i=1}^{q} r_{x_{i}}^{2} \nu^{*} \rightarrow \max _{b^{*}}
$$

Extrema of these functions are separately a method of multipliers of Lagrang [4]. Multipliers $\mu_{\mathrm{j}}^{*}, v_{\mathrm{j}}^{*}$ are interpreted as eigen values corresponding GDSP (6), (7). Necessary conditions of maxima of functions $\varphi_{2}, \varphi_{3}$ under the restriction (5) are equations:

$$
\begin{aligned}
& \left(\mathrm{R}_{12} \mathrm{R}_{21}-\mu_{\mathrm{j}}{ }^{*} \mathrm{R}_{11}\right) \mathrm{a}_{\mathrm{j}}{ }^{*}=0 \\
& \left(\mathrm{R}_{21} \mathrm{R}_{12}-v_{\mathrm{j}} \mathrm{R}_{22}\right) \mathrm{b}_{\mathrm{j}}{ }^{*}=0
\end{aligned}
$$

where $\mu_{j}{ }^{*}, j=1, \ldots, p$, are sets of eigen values for the corresponding sets eigen vectors $\mathbf{a}^{*}=\left(a^{*}{ }_{1 j}, \ldots, a^{*}{ }_{q j}\right)^{T}$, from matrix $\mathrm{A}_{\mathrm{qp}}^{*}=\left[\mathbf{a}_{1}^{*}\left|\mathbf{a}_{2}{ }_{2}\right| \ldots \mid \mathbf{a}_{\mathrm{p}}{ }^{*}\right]$, , and eigen values $v_{j}^{*}, j=1, \ldots, p$, correspond to a set eigen vectors $\mathbf{b}_{j}^{*}=\left(b^{*}{ }_{1 j}, \ldots, \quad b^{*}{ }_{p j}\right)^{\mathrm{T}}, \quad j=1, \ldots, p$, from matrix $\mathrm{B}_{\mathrm{pp}}^{*}=\left[\mathbf{b}^{*}{ }_{1}\left|\mathbf{b}_{2}{ }_{2}\right| \ldots \mid \mathbf{b}_{\mathrm{p}}{ }^{*}\right]$. Thus matrixes $\mathrm{U}^{*}{ }_{\mathrm{mp}} \mathrm{V}^{*}{ }_{\mathrm{mp}}$ redundancy $\mathrm{u}^{*}-$ and the $\mathrm{v}^{*}$-variables received accordingly from $x^{-}$and $y$-variables, satisfy to following equations:

$$
\begin{gathered}
U^{*}{ }_{m p}=Z_{1} A^{*}{ }_{p p}, V^{*}{ }_{m p}=Z_{2} B^{*}{ }_{p p}, \\
(1 / m) U^{* T} U^{*}=A^{* T} R_{11} A^{*}=I_{p p}, \\
(1 / m) V^{* T} V^{*}=B^{* T} R_{22} B^{*}=I_{p p}, \\
(1 / m) U^{* T} V^{*}=A^{* T} R_{12} B^{*}=\Psi_{12} \neq \Psi_{21} .
\end{gathered}
$$

Functions from squares of coefficients of correlations between variables were applied in multiple linear regression analysis (DM MLRA), RA. RA is applied to the forecast in [13]. Special cases of RA are $[3,14]$ multiple linear regression analysis (DM MLR) $\left(\mathrm{n}=\mathrm{q}+\mathrm{p}, \mathrm{p}=1\right.$, in DM MLRA $\mu_{\mathrm{j}}{ }^{*}=\mathrm{R}_{21} \mathrm{R}^{-1}{ }_{11} \mathrm{R}_{12}$ is solved DSP $\left.\left(R_{21} R_{12}-\mu_{j}{ }^{*} R_{11}\right) \mathbf{a}_{j}{ }_{j}=0\right)$ by $\mathbf{a}_{j}{ }_{j}=0$ ), PCA (direct model of the principal component analysis (DM of the PCA [6]), $n=q+p, p=0, Z_{1}=Z_{2}$ ). DSP from PCA $\left(R_{11}-\mu_{j}^{*} I\right) A^{*}=0_{p p}[15]$ is solved. Difference of RA from PCA that find a linear combination in RA from $q x$-variables ( $p$ y-variables) with coefficients $\mathbf{a}^{*}=\left(\mathrm{a}^{*}{ }_{1}, \ldots, \mathrm{a}_{\mathrm{q}}\right)^{\mathrm{T}}, \mathbf{b}^{*}=\left(\mathrm{b}^{*}{ }_{1}, \ldots, \mathrm{b}_{\mathrm{p}}\right)^{\mathrm{T}}$, which maximizes $\mathbf{a}^{* \mathrm{~T}} \mathrm{R}^{2} \mathbf{a}^{*}\left(\mathbf{b}^{* \mathrm{~T}} \mathrm{R}^{2} \mathbf{b}^{*}\right)$, where $\mathbf{a}^{* \mathrm{~T}} \mathrm{R} \mathbf{a}^{*}\left(\mathbf{b}^{* \mathrm{~T}} \mathrm{R} \mathbf{b}^{*}=1\right)$. In DM PCA (by $p=0$ ) find a linear combination of $n$ of $x-$ variables with coefficients $\mathbf{c}=\left(\mathrm{c}_{1}, \ldots, \mathrm{c}_{\mathrm{n}}\right)^{\mathrm{T}}$ which maximizes $\mathbf{c}^{\mathrm{T}} \mathrm{R} \mathbf{c}$, where $\mathbf{c}^{\mathrm{T}} \mathbf{c}=1$ [7]. At the same time the MLRAby $p=1$ represents a special case of CCA. At $\mathrm{Z}_{1}=\mathrm{Z}_{2}$ the equation from GDSP of the CCA contains a single matrix $\left(I_{n n}-\mu^{2} I_{n n}\right)=0$, that is possible in the presence of ideal coefficients of canonical correlation: $r_{u v}=1$. Therefore DM PCA is not a special case of CCA, and DM MLRA- yes. The numerical solution of problems (6), (7) is formally identical to calculations on a CCA, as matrix works $\mathrm{R}_{12} \mathrm{R}_{21}, \mathrm{R}_{21} \mathrm{R}{ }_{12}$ and matrixes $\mathrm{R}_{11}, \mathrm{R}_{22}$ - symmetric matrixes. However eigen values $\mu_{j}^{*}$ and $v_{j}^{*}$ in 2 GDSP different: $\mu_{j}^{*} \neq v_{j}^{*} j=1, \ldots, p$. So in RA there are 2 structures of eigen vectors of $\mathrm{A}_{\mathrm{qp}}^{*}$ and $\mathrm{B}_{\mathrm{pp}}^{*}$ :

$$
\mathrm{A}_{\mathrm{qp}}^{*}=\left[\mathrm{a}_{1}^{*}\left|\mathrm{a}_{2}{ }_{2}\right| \ldots \mid \mathrm{a}_{\mathrm{p}}^{*}\right], \mathrm{B}_{\mathrm{pp}}^{*}=\left[\mathrm{b}_{1}{ }_{1}\left|\mathrm{~b}_{2}{ }_{2}\right| \ldots \mid \mathrm{b}_{\mathrm{p}}^{*}\right] \text {. }
$$

In $\mathrm{RA}$ redundancy $\mathrm{u}^{*}$ - and $\mathrm{v}^{*}$-variables not biortogonality i.e. $\mathrm{u}^{*}$-set components not ortogonality to $\mathrm{v}^{*}$-set components $\Psi_{12} \neq \operatorname{diag}\left(\psi_{1}, \ldots, \psi_{\mathrm{p}}\right)$, for matrixes of eigen vectors $\mathrm{A}_{\mathrm{qp}}$ and $\mathrm{B}_{\mathrm{pp}}$ are defined separately (in CCA one of 2 matrixes of $p$ eigen 


\begin{tabular}{l|lr|ll|ll} 
& ISRA (India) & $=\mathbf{1 . 3 4 4}$ & SIS (USA) & $=\mathbf{0 . 9 1 2}$ & ICV (Poland) & $=\mathbf{6 . 6 3 0}$ \\
Impact Factor: & ISI (Dubai, UAE) $=\mathbf{0 . 8 2 9}$ & PUH (Russia) $=\mathbf{0 . 2 0 7}$ & PIF (India) & $=\mathbf{1 . 9 4 0}$ \\
& GIF (Australia) & $=\mathbf{0 . 5 6 4}$ & ESJI (KZ) & $=4.102$ & IBI (India) & $\mathbf{4 . 2 6 0}$ \\
& JIF & $=\mathbf{1 . 5 0 0}$ & SJIF (Morocco) & $\mathbf{2 . 0 3 1}$ & & \\
\hline
\end{tabular}

vectors $A_{q p}$ or $B_{p p}$ is calculated: if it is calculated $A_{q p}$, then $B_{p p}=\Lambda R_{21} A_{q p}$. Thus $u$ - and v-variables biortogonality: $\left.(1 / \mathrm{m}) \mathrm{U}^{\mathrm{T}} \mathrm{V}=\Lambda_{\mathrm{pp}}=\operatorname{diag}\left(\lambda_{1}, \ldots, \lambda_{\mathrm{p}}\right)\right)$.

\section{The redundancy formulas of the non-}

symmetrical redundancy of the $\bar{u}$ - and $\bar{v}$ variables in biorthogonal canonical-redundancy variables

To interpret squares of factorial loadings at initial $x$ - and $y$-variables from the CCA it is necessary to transform so $\mathrm{x}$ - and $\mathrm{y}$-variables that received new ( $\bar{u}$-and $\bar{v}$-) variables would become biorthogonal. Then squares of factorial loadings (squares of coefficients of correlations) at them will begin to be interpreted as determination coefficients at biorthogonal (in pairs uncorrelated) $\mathrm{j}$-th $\bar{u}$ and $\bar{v}$ - variables. CCA is for this purpose suitable. We subject two sets of redundancy $\mathrm{u}^{*}$-and $\mathrm{v}^{*}$-variables to biortogonalization transformation with CCA application. Then at redundancy-canonical variables we have two orthogonal matrixes $\bar{A}_{\mathrm{pp}}, \overline{\mathrm{B}}_{\mathrm{pp}}$, such that new matrixes $\bar{U}_{\mathrm{mp}}=\mathrm{U}^{*} \bar{A}_{\mathrm{pp}}, \quad \overline{\mathrm{V}}_{\mathrm{mp}}=\mathrm{V}^{*} \overline{\mathrm{B}}_{\mathrm{pp}}$ $\bar{u}$ - and $\bar{v}$-variables will be sets of biorthogonal redundancy and canonical variables. The solved spectral problem has already an appearance of a DSP

$$
\left(\Psi_{12} \Psi_{21}-\bar{\Lambda}^{2} \mathrm{I}\right) \bar{A}_{\mathrm{pp}}=\mathbf{0}_{\mathrm{pp}},
$$

because the corres-ponding GDSP

$$
\left(\Psi_{12} \Psi^{-1}{ }_{22} \Psi_{21}-\bar{\Lambda}{ }^{2} \Psi_{11}\right) \bar{A}=0_{\mathrm{pp}}
$$

because of existence of equalities

$\Psi_{11}=(1 / \mathrm{m}) \quad \mathrm{U}^{* \mathrm{~T}} \mathrm{U}^{*},=\mathrm{I} \quad \mathrm{pp}, \quad \Psi_{22}=(1 / \mathrm{m}) \mathrm{V}^{* \mathrm{~T}} \mathrm{~V}^{*}=\mathrm{I} \quad \mathrm{pp}$ becomes simpler and assumes DSP air:

$$
\left(\Psi_{12} \Psi_{21}-\bar{\Lambda}^{2}\right) \bar{A}=0
$$

We have to solve a problem (9) as GDSP from CCA, thus, having defined a matrix of eigen vectors $\bar{A}_{\mathrm{pp}}$, we define other matrix of eigen vectors

$\overline{\mathrm{B}}_{\mathrm{pp}}=\bar{\Lambda}^{-1} \Psi_{21} \bar{A}$. Here the diagonal matrix has an view $\bar{\Lambda}_{\mathrm{pp}}=\operatorname{diag}\left(\bar{\lambda}_{1}, \ldots, \bar{\lambda}_{\mathrm{p}}\right)$. Matrixes $\bar{U}_{\mathrm{mp}}, \overline{\mathrm{V}}_{\mathrm{mp}}$ of new biorthogonal variables satisfy to equations

$$
(1 / \mathrm{m}) \bar{U} \mathrm{~T} \bar{U}=\mathrm{I}_{\mathrm{pp}},(1 / \mathrm{m}) \overline{\mathrm{V}} \mathrm{T} \overline{\mathrm{V}}=\mathrm{I}_{\mathrm{pp}},
$$

(1/m) $\bar{U} \mathrm{~T} \overline{\mathrm{V}}=\bar{\Lambda}_{\mathrm{pp}}$, equivalent to equations:

$$
\bar{A} \cdot \Psi_{12} \overline{\mathrm{B}}=\bar{\Lambda}_{\mathrm{pp}}, \bar{A}^{\mathrm{T}} \overline{\boldsymbol{A}}=\mathrm{I}_{\mathrm{pp}}, \overline{\mathrm{B}}{ }^{\mathrm{T}} \overline{\mathrm{B}}=\mathrm{I}_{\mathrm{pp}} \text { (10) }
$$

It is geometrically possible to treat $\bar{A}_{\mathrm{pp}}$ and $\overline{\mathrm{B}}$ pp matrixes as the orthogonal matrixes of rotations containing sine and cosines of corners between old axes (for redundancy variables) and new axes (for biortogonal redundancy-canonical $\bar{u}$ and $\bar{v}$ variables).
Now we can consider redundancy of 4 pairs (from 8) sets of variables. Total the redundancy, caused by functional linear dependences, are equal 1 . For example, the redundancy of $\mathrm{p}$ y-variables caused by existence of $\mathrm{p} v$-variables, each of which linearly depends on $\mathrm{p} y$-variables, is equal 1 :

$$
\begin{gathered}
R_{\mathrm{p}}(\mathrm{Y}, \mathrm{V})=\sum_{i=1}^{p} \boldsymbol{R}_{p}\left(\boldsymbol{Y}, \boldsymbol{v}_{I}\right)=\sum_{i=1}^{p} \varphi_{2}\left(\boldsymbol{r}_{Y v_{i}}^{2}\right)= \\
=(1 / \mathrm{p}) \operatorname{tr}\left(\mathrm{B}^{\mathrm{T}} \mathrm{R}^{2}{ }_{22} \mathrm{~B}\right)=(1 / \mathrm{p}) \operatorname{tr}\left(\mathrm{R}_{22}\right)=(1 / \mathrm{p}) \mathrm{p}=1,
\end{gathered}
$$

redundancy of $\mathrm{q} x$-variables, $\mathrm{p}$ u-variables caused by existence, each of which linearly depends on $q$ of $x-$ variables, is equal 1:

$$
\begin{gathered}
R_{\mathrm{q}}(\mathrm{X}, \mathrm{U}) \sum_{i=1}^{p} \boldsymbol{R}_{q}\left(X, \boldsymbol{U}_{I}\right)=\sum_{i=1}^{p} \varphi_{3}\left(\boldsymbol{r}_{X u_{i}}^{2}\right)= \\
=(1 / \mathrm{q}) \operatorname{tr}\left(\mathrm{A}^{\mathrm{T}} \mathrm{R}^{2}{ }_{11} \mathrm{~A}\right)= \\
=(1 / \mathrm{q}) \operatorname{tr}\left(\mathrm{R}_{11}\right)=(1 / \mathrm{q}) \operatorname{tr}\left(\mathrm{R}_{11}\right)=(1 / \mathrm{q}) \mathrm{q}=1 .
\end{gathered}
$$

Other similar total indexes of redundancy $R_{\mathrm{q}}\left(\mathrm{X}, \mathrm{U}^{*}\right)$, $R_{\mathrm{p}}\left(\mathrm{Y}, \mathrm{V}^{*}\right), R_{\mathrm{q}}(\mathrm{X}, \bar{U}), R_{\mathrm{p}}\left(\mathrm{Y}, \overline{V)}, R_{\mathrm{p}}\left(\mathrm{U}^{*}, \bar{U}\right)\right.$,

$R_{\mathrm{p}}\left(\mathrm{V}^{*}, \bar{V}\right)$ are also equal 1 .

$$
\begin{aligned}
& R_{\mathrm{q}}(\mathrm{X}, \bar{U})=\sum_{i=1}^{q} \boldsymbol{R}\left(X, \overline{\boldsymbol{u}_{i}}\right)=\sum_{i=1}^{p} \varphi_{3}\left(\boldsymbol{r}_{x \bar{u}_{i}}^{2}\right)= \\
& =(1 / \mathrm{q}) \operatorname{tr}\left(\bar{A}{ }^{\mathrm{T}} \mathrm{A}^{* \mathrm{~T}} \mathrm{R}^{2}{ }_{11} \mathrm{~A}^{*} \bar{A}\right)= \\
& =(1 / \mathrm{q}) \operatorname{tr}\left(\bar{A}^{\mathrm{T}} \mathrm{I}_{\mathrm{qq}} \bar{A}=(1 / \mathrm{q}) \operatorname{tr}\left(\mathrm{I}_{\mathrm{qq}}\right)=(1 / \mathrm{q}) \mathrm{q}=1\right. \text {, } \\
& R_{\mathrm{p}}(\mathrm{Y}, \bar{V})=\sum_{i=1}^{p} \boldsymbol{R}_{p}\left(X, \overline{v_{i}}\right)=\sum_{i=1}^{p} \varphi_{2}\left(\boldsymbol{r}_{x \bar{v}_{i}}^{2}\right)= \\
& =(1 / \mathrm{p}) \operatorname{tr}\left(\overline{\mathrm{B}}{ }^{\mathrm{T}} \mathrm{B}^{* \mathrm{~T}} \mathrm{R}^{2}{ }_{22} \mathrm{~B}^{*} \overline{\mathrm{B}}\right)= \\
& =(1 / p) \operatorname{tr}\left(\overline{\mathrm{B}}{ }^{\mathrm{T}} \mathrm{I}_{\mathrm{pp}} \overline{\mathrm{B}}=(1 / \mathrm{p}) \operatorname{tr}\left(\mathrm{I}_{\mathrm{pp}}\right)=(1 / \mathrm{p}) \mathrm{p}=1\right. \text {, } \\
& R_{\mathrm{q}}\left(\mathrm{X}, \mathrm{U}^{*}\right)=\sum_{i=1}^{p} \varphi_{3}\left(\boldsymbol{r}_{X}^{2} u_{\boldsymbol{i}}\right)=(1 / \mathrm{q}) \operatorname{tr}\left(\mathrm{A}^{* \mathrm{~T}} \mathrm{R}^{2}{ }_{11} \mathrm{~A}^{*}\right)= \\
& =(1 / q) \operatorname{tr}\left(R_{11}\right)=(1 / q) q=1, \\
& R_{\mathrm{p}}\left(\mathrm{Y}, \mathrm{V}^{*}\right)=\sum_{i=1}^{p} \varphi_{2}\left(r_{Y}^{2} v_{i}^{*}\right)=(1 / \mathrm{p}) \operatorname{tr}\left(\mathrm{B}^{* \mathrm{~T}} \mathrm{R}^{2}{ }_{22} \mathrm{~B}^{*}\right)= \\
& =(1 / \mathrm{p}) \operatorname{tr}\left(\mathrm{R}_{22}\right)=(1 / \mathrm{p}) \mathrm{p}=1 \text {, } \\
& R_{\mathrm{p}}\left(\mathrm{U}^{*}, \bar{U}\right)=\sum_{i=1}^{p} \varphi_{2}\left(r_{u^{*} u_{\bar{i}}}^{2}\right)= \\
& =(1 / p) \operatorname{tr}\left(B^{* T} R^{2}{ }_{22} B^{*}\right)=(1 / p) \operatorname{tr}\left(R_{22}\right)=(1 / p) p=1 \text {, } \\
& R_{\mathrm{p}}\left(\mathrm{V}^{*}, \bar{V}\right)=\sum_{i=1}^{p} \varphi_{2}\left(\boldsymbol{r}_{V^{*}}^{2} v_{\bar{i}}\right)=
\end{aligned}
$$$$
=(1 / p) \operatorname{tr}\left(B^{* T} R^{2}{ }_{22} B^{*}\right)=(1 / p) \operatorname{tr}\left(R_{22}\right)=(1 / p) p=1 .
$$

At a conclusion of some of these formulas ratios (11) were used formulas [4]:

$$
\mathrm{R}_{11} \mathrm{AA}^{\mathrm{T}} \mathrm{R}_{11}=\mathrm{R}_{11}, \mathrm{R}_{22} \mathrm{BB}^{\mathrm{T}} \mathrm{R}_{22}=\mathrm{R}_{22}
$$

Besides, redundancy $\varphi_{1}\left(r^{2}{ }_{u v}\right)$ to $u$-variable in the presence of a $v$-variable is equal in the CCA to redundancy $\varphi_{1}\left(\mathrm{r}_{\mathrm{vu}}^{2}\right)$ to $\mathrm{v}$-variable in the presence of a u-variable: $\quad \varphi_{1}\left(\mathrm{r}_{\mathrm{uv}}^{2}\right)=\varphi_{1}\left(\mathrm{r}_{\mathrm{vu}}^{2}\right)=\lambda^{2}$. To redundancy of 


\begin{tabular}{l|lr|ll|ll} 
& ISRA (India) & $=\mathbf{1 . 3 4 4}$ & SIS (USA) & $=\mathbf{0 . 9 1 2}$ & ICV (Poland) & $=\mathbf{6 . 6 3 0}$ \\
Impact Factor: & ISI (Dubai, UAE) $=\mathbf{0 . 8 2 9}$ & PUH (Russia) $=\mathbf{0 . 2 0 7}$ & PIF (India) & $=\mathbf{1 . 9 4 0}$ \\
& GIF (Australia) & $=\mathbf{0 . 5 6 4}$ & ESJI (KZ) & $=4.102$ & IBI (India) & $\mathbf{4 . 2 6 0}$ \\
& JIF & $=\mathbf{1 . 5 0 0}$ & SJIF (Morocco) & $\mathbf{2 . 0 3 1}$ & & \\
\hline
\end{tabular}

two sets of $\mathrm{u}$ - and v-variables of initial variables are symmetric: $R_{\mathrm{p}}(\mathrm{U}, \mathrm{V})=R_{\mathrm{p}}(\mathrm{V}, \mathrm{U})=\sum_{j=1}^{p} \lambda_{j}^{2}=\max _{1}$

Total redundancy of $\mathrm{q} x$-variables is not equal in the CCA to total redundancy of $\mathrm{p} y$-variables:

$$
\mathrm{R}_{\mathrm{q}}(\mathrm{X}, \mathrm{Y}) \neq \mathrm{R}_{\mathrm{p}}(\mathrm{Y}, \mathrm{X}), \mathrm{q} \neq \mathrm{p} \text {. }
$$

In a CCA indexes of redundancy maximized separately. Therefore are not maximized total redundancy. Other total redundancy is maximized $\mathrm{R}_{\mathrm{p}}(\mathrm{U}, \mathrm{V}), \mathrm{R}_{\mathrm{p}}(\mathrm{V}, \mathrm{U})$.

Theorem 1. In a CCA total redundancy of initial $\mathrm{x}^{-}$and $\mathrm{y}$-variables are equal each other and equal to total redundancy of canonical $\mathrm{u}-$ and $\mathrm{v}-$ variables.

$$
\begin{gathered}
(1 / \mathrm{p}) \sum_{j=1}^{p} \lambda_{j}^{2}=R_{\mathrm{p}}(\mathrm{V}, \mathrm{U})=R_{\mathrm{p}}(\mathrm{Y}, \mathrm{U})=R_{\mathrm{q}}(\mathrm{X}, \mathrm{V})= \\
=R_{\mathrm{p}}(\mathrm{U}, \mathrm{V})
\end{gathered}
$$

Proof. By definition $R_{p}(Y, u)=(1 / p) \quad \mathbf{r}_{Y u}^{T} \mathbf{r}_{Y u}$. Therefore

$$
\begin{gathered}
R_{\mathrm{p}}(\mathrm{Y}, \mathrm{U})=\sum_{i=1}^{p} R_{p}\left(Y, u_{I}\right)=\sum_{i=1}^{p}(1 / \mathrm{p}) \mathbf{r}^{\mathrm{T}} \mathrm{Yu}_{\mathrm{Yu}}= \\
\left.=\sum_{i=1}^{p}\left(\mathrm{R}_{21} \mathrm{a}\right)^{\mathrm{T}} \mathrm{R}_{21} \mathrm{a}\right)=\sum_{i=1}^{p} \mathrm{a}^{\mathrm{T}} \mathrm{R}_{12} \mathrm{R}_{21} \mathrm{a}= \\
=(1 / \mathrm{p}) \sum_{i=1}^{p}\left(v \mathrm{R}_{22} \mathbf{b}\right)^{\mathrm{T}}\left(v \mathrm{R}_{22} \mathbf{b}\right)=(1 / \mathrm{p}) \sum_{i=1}^{p} v^{2} \mathbf{r}^{\mathrm{T}} \mathrm{Yv}_{\mathrm{V}} \mathbf{r}_{\mathrm{Yv}}= \\
=\sum_{i=1}^{p} v^{2} R_{\mathrm{p}}(\mathrm{Y}, \mathrm{v})=\sum_{i=1}^{p} v^{2}(1 / \mathrm{p})=\sum_{i=1}^{p} \lambda^{2}(1 / \mathrm{p})= \\
=R_{\mathrm{p}}(\mathrm{U}, \mathrm{V})
\end{gathered}
$$

Here equalities $\mathrm{R}_{21} \mathrm{a}=v_{\mathrm{pp}} \mathrm{R}_{22}$ and $R_{\mathrm{p}}(\mathrm{Y}, \mathrm{v})=1 / \mathrm{p}$ was applied. Equality $R_{\mathrm{q}}(\mathrm{X}, \mathrm{V})=\sum_{j=1}^{p} \mu_{j}^{2} R_{\mathrm{p}}\left(\mathrm{X}, \mathrm{u}_{\mathrm{j}}\right)$ is similarly prove. Using definition $R_{\mathrm{q}}(\mathrm{X}, \mathrm{v})=$ $=(1 / q) \operatorname{tr}\left(b^{T} R_{21} R_{12} b\right)$ and a formula $R_{12} b=\mu R_{11} a$ we have

$$
\begin{gathered}
R_{\mathrm{q}}(\mathrm{X}, \mathrm{V})=\sum_{i=1}^{p} \varphi_{3}\left(\mathrm{r}^{2}{ }_{\mathrm{Xv}}\right)==\sum_{i=1}^{p} R_{\mathrm{q}}(\mathrm{X}, \mathrm{v})= \\
=\sum_{i=1}^{p}(1 / \mathrm{q}) \mathbf{r}^{\mathrm{T}} \mathrm{Xv}_{\mathrm{V}} \mathbf{r}_{\mathrm{Xv}}=\sum_{i=1}^{p}(1 / \mathrm{q})\left(\mathrm{R}_{12} \mathbf{b}\right)^{\mathrm{T}}\left(\mathrm{R}_{12} \mathbf{b}\right)= \\
=\sum_{i=1}^{p}(1 / \mathrm{q})\left(\mu \mathrm{R}_{11} \mathbf{a}\right)^{\mathrm{T}}\left(\mu \mathrm{R}_{11} \mathbf{a}\right)= \\
=\sum_{i=1}^{p}(1 / \mathrm{q}) \mu^{2} \mathbf{r}^{\mathrm{T}}{ }_{\mathrm{Xu}} \mathbf{r}_{\mathrm{Xu}}=\sum_{i=1}^{p} \mu^{2} R_{\mathrm{q}}(\mathrm{X}, \mathrm{u}) .
\end{gathered}
$$

Owing to linear functional dependence of $\mathrm{u}$ variable from each of $\mathrm{q} x$-variables, is followed that by the share of dispersion explained with linear dependence on $\mathrm{q} x$-variables, the is equal $1 / \mathrm{p}$, to $\mathrm{j}=1, \ldots, \mathrm{p}$, i.e. $R_{s}\left(X, u_{I}\right)=1 / \mathrm{p}$, we have demanded equality:

$$
R_{\mathrm{p}}(\mathrm{Y}, \mathrm{U})=R_{\mathrm{q}}(\mathrm{X}, \mathrm{V})=\sum_{j=1}^{p} \lambda_{j}^{2}(1 / \mathrm{p})=R_{\mathrm{p}}(\mathrm{U}, \mathrm{V})
$$

Having equated the right parts of equalities (16), (17) we have required equality (15). Then

$$
R_{\mathrm{q}}(\mathrm{X}, \mathrm{V})=\sum_{j=1}^{p} \mu_{j}^{2} R_{q}\left(X, u_{j}\right)=\sum_{j=1}^{p} \mu_{j}^{2}(1 / \mathrm{p})=R_{\mathrm{p}}(\mathrm{V}, \mathrm{U})
$$

Ratio between the redundancy, similarly considered in the Theorem 1, we have when transforming by the $\mathrm{CCA}$ of matrixes of $\mathrm{U}^{*}{ }_{\mathrm{mp}}, \mathrm{V}^{*}{ }_{\mathrm{mp}}$ redundancy variables.

We will consider redundancy of the new variables received at consecutive transformation of initial variables by two specified methods. When transforming by RA are maximized all $\mathrm{p}$ pairs of redundancy:

$R_{\mathrm{P}}\left(\mathrm{Y}, \mathrm{u}_{\mathrm{j}}{ }^{*}\right)=\varphi_{2}\left(r_{Y u_{i}}^{2}\right)=(1 / \mathrm{p}) \mathbf{b}_{\mathrm{j}}{ }^{* \mathrm{~T}} \mathrm{R}_{21} \mathrm{R}_{12} \mathbf{b}_{\mathrm{j}}{ }^{*}=(1 / \mathrm{p}) \mu_{\mathrm{j}}{ }^{* 2}$

$R_{\mathrm{q}}\left(\mathrm{X}, \mathrm{v}_{\mathrm{j}}^{*}\right)=\varphi_{3}\left(\boldsymbol{r}_{X v_{i}}^{2}\right)=(1 / \mathrm{q}) \mathbf{a}^{* \mathrm{~T}} \mathrm{R}_{12} \mathrm{R}_{21} \mathbf{a}_{\mathrm{j}}^{*}=(1 / \mathrm{q}) v^{* 2}{ }_{\mathrm{j}}$

Therefore, are maximized total redundancy:

$$
\begin{gathered}
R_{\mathrm{P}}\left(\mathrm{Y}, \mathrm{U}^{*}\right)=\sum_{i=1}^{p} \varphi_{2}\left(\boldsymbol{r}_{\left.Y u_{i}\right)=}^{2}{ }^{p} \sum_{i=1}^{p} \mathbf{b}_{\mathrm{j}}{ }^{* \mathrm{~T}} \mathrm{R}_{21} \mathrm{R}_{12} \mathbf{b}_{\mathrm{j}}{ }^{*}=(1 / \mathrm{p}) \sum_{i=1}^{p} \mu_{\mathrm{j}}{ }^{* 2}=\max _{2}\right. \\
R_{\mathrm{q}}\left(\mathrm{X}, \mathrm{V}^{*}\right)=\sum_{i=1}^{p} \varphi_{2}\left(\boldsymbol{r}_{\left.X v_{i}\right)=}^{2}\right. \\
=(1 / \mathrm{q}) \sum_{i=1}^{p} \mathbf{a}_{\mathrm{j}}{ }^{* \mathrm{~T}} \mathrm{R}_{12} \mathrm{R}_{21} \mathbf{a}_{\mathrm{j}}{ }^{*}=(1 / \mathrm{q}) \sum_{i=1}^{p} v_{\mathrm{j}}{ }^{* 2}=\max _{3}
\end{gathered}
$$

In the redundancy analysis we look for a linear combination from $x, a^{*}$ which maximizes $\mathbf{a}^{* T} \mathrm{R}^{2}{ }_{11} \mathbf{a}^{*}$, where $\mathbf{a}^{* \mathrm{~T}} \mathrm{R}_{11} \mathbf{a}^{*}=1, \mathbf{a}^{*}-\mathrm{j}$-th eigen vector. When transforming a $\mathrm{m} \times 2 \mathrm{p}$-matrix $\left[\mathrm{U}^{*}{ }_{1} \mathrm{~V}^{*}\right]$ of redundancy variables by the CCA (it is made on one of method steps from [8]) are maximized redundancy:

$$
\begin{gathered}
R_{\mathrm{p}}\left(\mathrm{U}^{*}, \bar{v}_{\mathrm{i}}\right)=\varphi_{2}\left(\mathbf{r}^{2}\left(\mathrm{U}^{*}, \bar{v}_{\mathrm{i}}\right)=\bar{\lambda}^{2} \times \varphi_{2}\left(\mathbf{r}^{2}\left(\mathrm{U}^{*}, \overline{\mathrm{u}}\right)=\bar{\lambda}^{2}\right.\right. \\
(1 / \mathrm{p}), \\
R_{\mathrm{p}}\left(\mathrm{V}^{*}, \overline{\mathrm{u}}_{\mathrm{i}}\right)=\varphi_{2}\left(\mathbf{r}^{2}\left(\mathrm{~V}^{*}, \overline{\mathrm{u}}_{\mathrm{i}}\right)=\right. \\
=\bar{\lambda}^{2} \times \varphi_{2}\left(\mathbf{r}^{2}\left(\mathrm{~V}^{*}, \bar{v}_{\mathrm{i}}\right)=\bar{\lambda}^{2}(1 / \mathrm{p}) \rightarrow \max ,\right.
\end{gathered}
$$

or it is maximized

$$
\begin{gathered}
\varphi_{1}\left(\mathrm{r}^{2}(\bar{u}, \bar{v})=\bar{\lambda}^{2} \text { and } \varphi_{2}\left(\boldsymbol{r}_{u^{*} u}^{2}\right)=(1 / \mathrm{p}) \bar{a}^{\mathrm{T}} \bar{a}=\right. \\
=(1 / \mathrm{p}) 1=1 / \mathrm{p},
\end{gathered}
$$




\begin{tabular}{l|lr|ll|ll} 
& ISRA (India) & $=\mathbf{1 . 3 4 4}$ & SIS (USA) & $=\mathbf{0 . 9 1 2}$ & ICV (Poland) & $=\mathbf{6 . 6 3 0}$ \\
Impact Factor: & ISI (Dubai, UAE) $=\mathbf{0 . 8 2 9}$ & PUHЦ (Russia) $=\mathbf{0 . 2 0 7}$ & PIF (India) & $=\mathbf{1 . 9 4 0}$ \\
& GIF (Australia) & $\mathbf{0 . 5 6 4}$ & ESJI (KZ) & $=\mathbf{4 . 1 0 2}$ & IBI (India) & $=\mathbf{4 . 2 6 0}$ \\
& JIF & $=\mathbf{1 . 5 0 0}$ & SJIF (Morocco) & $=\mathbf{2 . 0 3 1}$ & & \\
\hline
\end{tabular}

$$
\varphi_{2}\left(r_{v^{*} v_{i}}^{2}\right)=(1 / \mathrm{p}) \bar{b}^{\mathrm{T}} \bar{b}=(1 / \mathrm{p}) 1=1 / \mathrm{p} . \text { As } \bar{\lambda}^{2}<1
$$

then $\varphi_{2}\left(\mathbf{r}^{2}\left(\mathrm{u}^{*}, \bar{v}_{\mathrm{i}}\right)\right)<\varphi_{2}\left(\mathbf{r}^{2}\left(\mathrm{u}^{*}, \overline{\mathrm{u}}_{\mathrm{i}}\right)\right)$,

$$
\varphi_{2}\left(\mathbf{r}^{2}\left(\mathrm{v}^{*}, \overline{\mathrm{u}}_{\mathrm{i}}\right)\right)<\varphi_{2}\left(\mathbf{r}^{2}\left(\mathrm{v}^{*}, \bar{v}_{\mathrm{i}}\right)\right) .
$$

Maximum total redundancis of $\mathrm{u}^{*}$-and $\mathrm{v}^{*}$-variables are equal each other to

$$
R_{\mathrm{p}}\left(\mathrm{U}^{*}, \bar{V}\right)=R_{\mathrm{p}}\left(\mathrm{V}^{*}, \bar{U}\right)=\bar{\lambda}^{2}(1 / \mathrm{p})=\max _{4}
$$

If in the Theorem 1 instead of $\mathrm{x}$ - and $\mathrm{y}$-variables to consider $\mathrm{u}^{*}$-and $\mathrm{v}^{*}$-variables that equality (15) it will be transformed to equality (18).

Theorem 2. When transforming redundancy $u^{*}-$ and $\mathrm{v}^{*}$-variables to CCA maximized total redundancy of $\mathrm{x}$ - and $\mathrm{y}$-variables don't change

$$
\begin{aligned}
& R_{\mathrm{p}}(\mathrm{Y}, \bar{U})=R_{\mathrm{q}}\left(\mathrm{Y}, \mathrm{U}^{*}\right)=(1 / \mathrm{p}) \sum_{j=1}^{p} \mu_{j}^{* 2}=\max _{2}, \\
& R_{\mathrm{q}}\left(\mathrm{X}, \overline{V)}=R_{\mathrm{q}}\left(\mathrm{X}, \mathrm{V}^{*}\right)=(1 / \mathrm{q}) \sum_{i=1}^{p} v^{* 2}{ }_{\mathrm{j}}=\max _{3} .\right.
\end{aligned}
$$

Proof. When transforming redundancy of $\mathrm{u}^{*}-$ and $\mathrm{v}^{*}-$ variables to CCA total redundancy of the $\mathrm{p} \mathrm{y} \mathrm{-}$ variables, $\mathrm{p} \bar{u}$-variables caused by existence, are equal

$$
\begin{aligned}
& R_{\mathrm{p}}(\mathrm{Y}, \bar{U})=(1 / \mathrm{p}) \operatorname{tr}\left(\bar{A}^{\mathrm{T}} \mathrm{A}^{* \mathrm{~T}} \mathrm{R}_{12} \mathrm{R}_{21} \mathrm{~A}^{*} \bar{A}\right)= \\
& =(1 / \mathrm{p}) \operatorname{tr}\left(\bar{A}^{\mathrm{T}} \mu^{* 2}{ }_{\mathrm{pp}} \bar{A}\right)=(1 / \mathrm{p}) \operatorname{tr}\left(\mu_{\mathrm{pp}}^{* 2}\right)= \\
& =(1 / \mathrm{p}) \sum_{j=1}^{p} \mu_{j}^{* 2}=R_{\mathrm{p}}\left(\mathrm{Y}, \mathrm{U}^{*}\right)=\max _{2} .
\end{aligned}
$$

Similarly total redundancy of the q x-variables, $\mathrm{p} \bar{v}$ variables caused by existence, is equal

$$
\begin{aligned}
& R_{\mathrm{p}}(\mathrm{X}, \bar{V})=(1 / \mathrm{p}) \operatorname{tr}\left(\bar{B}{ }^{\mathrm{T}} \mathrm{B}^{* \mathrm{~T}} \mathrm{R}_{21} \mathrm{R}_{12} \mathrm{~B}^{*} \bar{B}\right)= \\
& \quad=(1 / \mathrm{p}) \operatorname{tr}\left(\bar{B}^{\mathrm{T}} v^{* 2}{ }_{\mathrm{pp}} \bar{B}\right)=(1 / \mathrm{p}) \operatorname{tr}\left(v^{* 2} \mathrm{pp}\right)=
\end{aligned}
$$

$$
=(1 / \mathrm{p}) \sum_{j=1}^{p} v_{j}^{2}=R_{\mathrm{p}}\left(\mathrm{X}, \mathrm{V}^{*}\right)=\max _{3}
$$

The theorem 2 is proved.

\section{Conclusion}

To us Theorems 1, 2 are useful. From the Theorem 1 follows that the CCA doesn't maximize the relations necessary to use. From the Theorem 2 follows that the RA maximizes separately relations between $\mathrm{x}-$ and $\mathrm{v}^{*}$-variables and relations between $\mathrm{y}$ andu*-variables:

$$
\begin{aligned}
& R_{\mathrm{q}}\left(\mathrm{X}, \mathrm{V}^{*}\right)=\max _{3}, R_{\mathrm{q}}\left(\mathrm{Y}^{\mathrm{Y}} \mathrm{U}^{*}\right)=\max _{3}, \\
& R_{\mathrm{p}}\left(\mathrm{U}^{*}, \bar{V}\right)=R_{\mathrm{p}}\left(\mathrm{V}^{*}, \bar{U}\right)=\bar{\lambda}^{2}(1 / \mathrm{p})=\max _{4}
\end{aligned}
$$

After transformation of excess $\mathrm{u}^{*}$ - and $\mathrm{v}^{*}$ variables the CCA maximizes relations between new $\overline{\mathrm{U}}$ - and $\overline{\mathrm{V}}$-variables. Before $\mathrm{u}^{*}$-variables were strongly related with y-variables, and $v^{*}$-variables with $\mathrm{x}$-variables. Thus take place of equalities (18), (19) i.e. degrees of interrelations between group $x$ and $\mathrm{v}^{*}$-variable and between group $\mathrm{y}$ - and $\mathrm{u}^{*}-$ variables have the same maximum value. In other words, the RA maximizes on the average factorial loadings at $\mathrm{x}$ - and $\mathrm{y}$-variables, and the subsequent transformations of excess variables by the CCA doesn't change the value of extent of relation $\mathrm{x}$ - and $y$-variables with their new factors, but does diagonal a correlacion matrix of these factors. This diagonalization reduces number of the parameters characterizing interrelations between factors, with $\mathrm{p}^{2}$ to $\mathrm{p}$. It, in turn, simplifies ratios between values initial $x-$ and $y$-variables and factors, the formula of an assessment of values of one variables is brought out of these ratios on values of other variables. Theoretical prerequisites for a method of predictive variables [8].

\section{References:}

1. Zhanatauov SU (2017) Block-diagonal correlation matrices of $\lambda$-samples. International scientific journal Theoretical \&Applied Science. 2017,№12,vol.56, p.101-111.

2. Hotelling H. (1936) Relations between two sets of variates.Biometrika, 1936, №28 (3-4): p.321377.

3. Krayako M. (1982) Canonical analysis.Biometr. J., 1982, vol. 24,№ 3, p. 211-228.
4. Van den Vollenberg A.L. (1977) Redundancy analysis - an alternative for canonical correlation analysis.- Psychometrika, 1977, vol.42, № 26, p. 207-219.

5. Thissen, M. ,Van den Wollenberg, A.L. (1975) REDANAL. A FORTRAN IV G/H program for redundancy analysis (Research Bulletin 26). Nijmegen, the Neterlan-ds: University of Nijmegen, Department of Mathematical Psychology, 1975. 


\begin{tabular}{l|lr|ll|ll} 
& ISRA (India) & $=\mathbf{1 . 3 4 4}$ & SIS (USA) & $=\mathbf{0 . 9 1 2}$ & ICV (Poland) & $=\mathbf{6 . 6 3 0}$ \\
Impact Factor: & ISI (Dubai, UAE) $=\mathbf{0 . 8 2 9}$ & PUHЦ (Russia) $=\mathbf{0 . 2 0 7}$ & PIF (India) & $=\mathbf{1 . 9 4 0}$ \\
& GIF (Australia) & $\mathbf{0 . 5 6 4}$ & ESJI (KZ) & $=4.102$ & IBI (India) & $=\mathbf{4 . 2 6 0}$ \\
& JIF & $=\mathbf{1 . 5 0 0}$ & SJIF (Morocco) & $=\mathbf{2 . 0 3 1}$ & & \\
\hline
\end{tabular}

6. Stewart D., Love W. A. (1968) general canonical correlation index.- Psychological Bulletin,1968.vol.70, p.160-163.

7. Zhanatauov S.U. (1987) Obratnaya model' glavnykh komponent i ee primenenie. Diss. na soiskanie uch. step.. kand. fiz.-mat. nauk:05.13.11:zashchishchena 8.12.1987: utv. 1.06.1988/Zhanatauov Sapargali UtepovichVychislitel'nyy tsentr Sibirs kogo otdeleniya AN SSSR, Novosibirsk, 1987g.,302 p.

8. Zhanatauov S.U. (2013) Obratnaya model' glavnykh komponent:-monografiya. -Almaty: Kazstatinform, 2013.- 201 p.

9. Zhanatauov S.U. (2017) A model of calculation risk changing of the interest rate "yield to maturity date" for foreign currency bonds of the republic of Kazakhstan. International scientific journal Theoretical\&AppliedScience. 2017, №8,vol.52,rr.19-36.http:// www.T-Science.org .

10. Zhanatauov SU. (2017) Theorem on the $\Lambda$ samples. International scientific journal Theoretical \&Applied Science.2017,№ 9, vol.53, p.177-192. http://www.T-Science.Org.

11. Zhanatauov SU. (2017) Optimization problem of modeling missing elements of the spectrum of the correlation matrix. International scientific journal Theoretical\&Applied Science. 2017, №10,vol.54, p.189-198.

12. Zhanatauov SU (2017) The optimization problem with linearized equations f-parameters (f1,f2,f3,f4,f5,f6)-spectrum. International scientific journal Theoretical \&Applied Science. 2017,№11,vol.55, p.251-267.

13. Zhanatauov S.U. (1981) Metod prognosticheskikh peremennykh.V knige «Vychislitel' nye sistemy» IM SO AN SSSR, 1981g. № 88, p. 151-155.

14. Oliveira M.R., Branco J.A., Croux C., Filzmoser P. (2004) Robust redundancy analysis by alternating regression. In $\mathrm{M}$. Hubert, G. Pison, A. Struyf and S. Van Aelst, editors, Theory and Applications of Recent Robust Methods, Series: Statistics for Industry and Technology, p. 235-246, Birkhauser, Basel, 2004.

15. Hotelling H. (1933) Analysis of a complex of statistical variables into principal compo nents. -J. Educ. Psychol.,1933, vol.24, p. 417-441, p. 498-520. 